\title{
Inner radio structure of compact BAL quasar $1045+352$
}

\section{Magdalena Kunert-Bajraszewska*}

Toruń Centre for Astronomy, N. Copernicus University, 87-100 Toruń, Poland

E-mail: magda@astro.uni.torun.pl

\section{Marcin P. Gawroński}

Toruń Centre for Astronomy, N. Copernicus University, 87-100 Toruń, Poland

E-mail: motylek@astro.uni.torun.pl

The first multifrequency radio observations of the very compact BAL quasar, 1045+352, were made using MERLIN and the VLBA in a snapshot mode. However, its unusual radio structure was still very difficult to interpret, indicating a scenario of intermittent activity or a jet precession. Here, we present some of a new full-track radio observations of 1045+352 made with the EVN+MERLIN at $5 \mathrm{GHz}$. The new more sensitive high-resolution observations made possible to trace the connection between the arcsecond structure and the radio core, and showed the presence of strong interactions between the jet and the medium of the host galaxy.

The 9th European VLBI Network Symposium on The role of VLBI in the Golden Age for Radio Astronomy and EVN Users Meeting

September 23-26, 2008

Bologna, Italy

\footnotetext{
* Speaker.
} 


\section{Introduction}

Broad absorption lines (BALs) are seen in 10-30\% (depends on the selection criteria) of both the radio-quiet and radio-loud quasar populations. They are probably caused by the outflow of gas with high velocities and are a part of the accretion process. There are two scenarios explaining origin and nature of BAL quasars. According to first, BAL regions exist in both BAL and nonBAL quasars, and the BAL quasars are normal quasars seen along a particular line of sight [2]. The second says that BALs may be associated with an evolutionary phase with a large BAL wind covering fraction, rather than orientation [3]. The radio morphologies of radio-loud BAL quasars provide important additional information about their orientation and the direction of the outflow, but only a handful of them have been resolved using radio observations. Most of those discovered to date are compact at radio frequencies with either a flat or steep spectrum, suggesting a wide range of orientation.

It has been suggested [1] that, because of their small sizes, most of the radio-loud BAL quasars belong to the class of compact radio sources, named compact steep spectrum (CSS) objects and gigahertz peaked spectrum (GPS) objects. GPS and CSS sources are considered to be young radio sources with linear sizes less then $20 \mathrm{kpc}$, entirely contained within the extent of the host galaxy.

There are only a few radio-loud compact BALQSOs with resolved structures and only at one frequency $[4,7]$. The first multifrequency radio observations of very compact BAL quasar $1045+352$ were made by our group [5]. Since the radio structure of 1045+352 appeared to be very complex and difficult to interpret based on the obtained images, we have made new high resolution and high sensitive EVN+MERLIN $5 \mathrm{GHz}$ observations of this source. Here we present some of the results of the new observations, the details of which will be discussed in separate paper.

\section{Observations and preliminary results}

$1045+352$ is a quasar with a redshift of $z=1.604$ [9] at $\mathrm{RA}=10^{\mathrm{h}} 48^{\mathrm{m}} 34^{\mathrm{s}} 247, \mathrm{Dec}=+34^{\mathrm{o}} 57^{\prime \prime} 24^{\prime} 99$ (coordinates for J2000 extracted from FIRST). It is a CSS object and its largest linear size is equal to $4.3 \mathrm{kpc}\left(\mathrm{H}_{0}=71 \mathrm{~km} \mathrm{~s}^{-1} \mathrm{Mpc}^{-1}, \Omega_{M}=0.27, \Omega_{\Lambda}=0.73\right) .1045+352$ has a very reddened spectrum showing a high-ionisation C IV broad absorption system [9], so the source has been clasified as a high-ionisation broad absorption line (HiBAL) quasar.

$1045+352$ belongs to the primary sample of 60 candidates for CSS sources selected from the VLA FIRST catalogue [8]. Initial observations of all the candidates were made with MERLIN at $5 \mathrm{GHz}$ [6] and 1.7, 5 and 8.4-GHz VLBA follow-up of 1045+352 was carried out on 13 November 2004 in a snapshot mode with phase-referencing [5]. The new images showed that this source have a complex radio morphology with a radio jet axis reorientation, that may result from (1) a merger, (2) a jet precession, or (3) jet-cloud interactions. However, to indicate one of the above-mentioned scenarios we planned more sensitive high-resolution EVN+MERLIN $5 \mathrm{GHz}$ observations that was carried out on 2 June 2007 in a full-track mode with phase-referencing. The target source scan was interleaved with a scan on a phase reference source and the total cycle time (target and phasereference) was $\sim 7$ minutes including telescope drive times, with $\sim 4$ minutes actually on the target source per cycle. The whole data reduction process was carried out using standard AIPS procedures. For the target source, the corresponding phase-reference source was mapped and the phase 

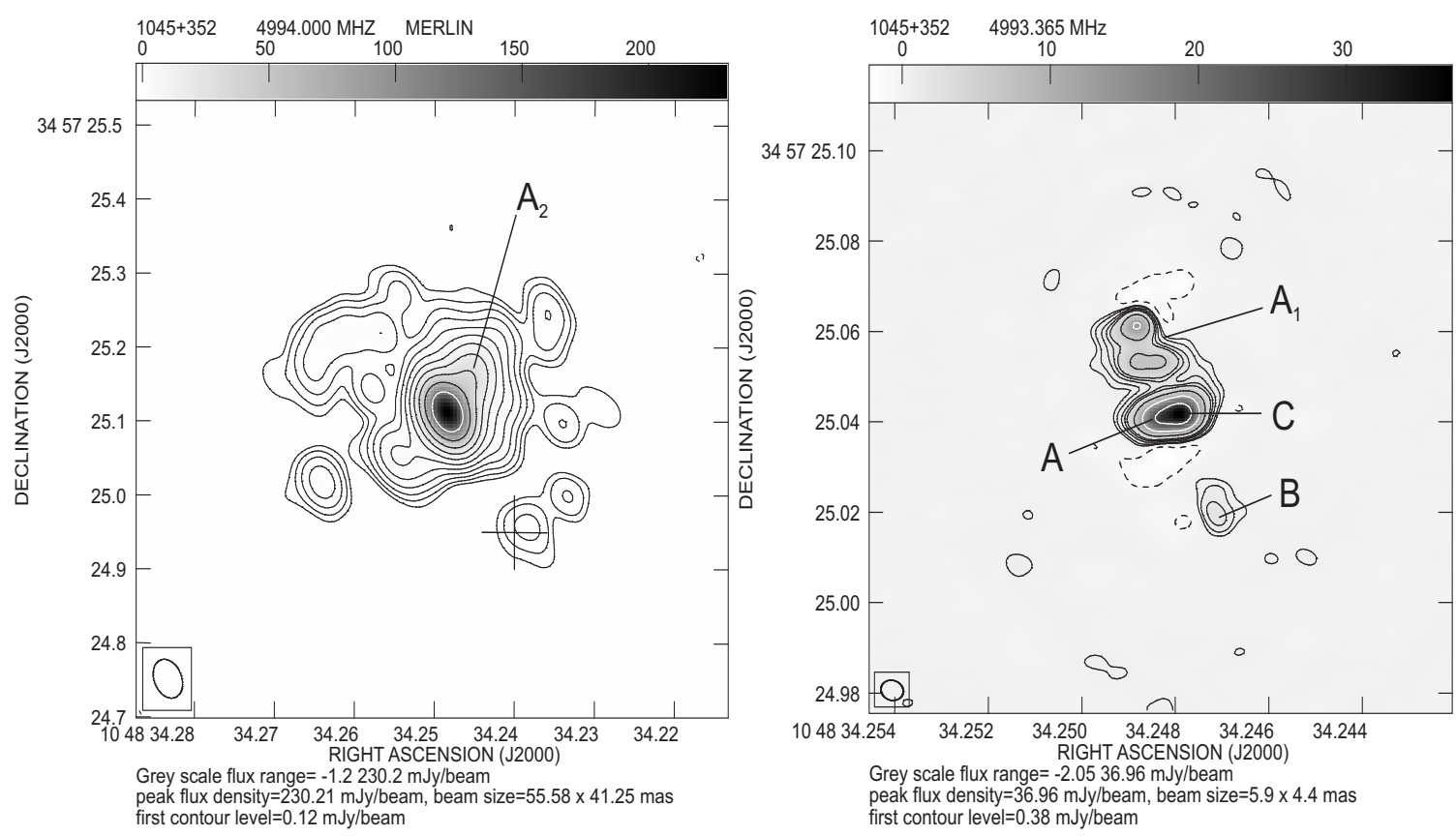

Figure 1: The $5 \mathrm{GHz}$ MERLIN image from [5] and the new $5 \mathrm{GHz}$ EVN image of $1045+352$. Contours increase by a factor 2 and the first contour level corresponds to $\approx 3 \sigma$. A cross indicates the position of an optical object found using the SDSS.

errors so determined were applied to the target source, which were then mapped using a few cycles of phase self-calibration and imaging. IMAGR was used to produce the final images separetely for EVN and MERLIN, and finally the combined EVN+MERLIN image was created. Here, we present preliminary $5 \mathrm{GHz}$ EVN image of 1045+352 (Fig. 1).

The new $5 \mathrm{GHz}$ EVN observations showed a jet (indicated as a A, Fig. 1) coming from the core (C) in a E/SE direction, and then another jet $\left(A_{1}\right)$ emerging in a NE direction. The feature indicated as a $\mathrm{B}$ is probably a counter-jet, and a trace of it was also visible in a previous lower resolution 1.7GHz VLBA image [5]. There is an appreciable $\left(\sim 60^{\circ}\right)$ misalignment between the axis of the inner structure (jet $\mathrm{A}$ ) with respect to the outer structure (jet $A_{1}$ ). We suggest the following interpretation of the radio structure of $1045+352$. The radio jet emerging from the core in a E/SE direction is not able to get through the dense environment and bents to a NE direction (component $A_{1}$ ). It's continuation $\left(A_{2}\right)$ is visible on MERLIN $5 \mathrm{GHz}$ image (Fig. 1). The weakness of the counter-jet emission is probably caused by the large beaming. We suspect the angle between the jet axis and the observer is small, much less than the $30^{\circ}$ we estimated last time [5].

Actually there are 17 compact BAL quasars observed with VLBI in a literature $[4,5,7]$ [and Montenegro-Montes' talk and proceeding]. About half of them have still unersolved structures even at high resolution, the other have core-jet structures indicating some orientation or very complex morphology, suggesting at least strong interaction with the surrounding medium [5].

\section{Summary}

$1045+352$ is a CSS object and a HiBAL quasar with a medium redshift. The new more sensi- 
tive high-resolution EVN $5 \mathrm{GHz}$ observations revealed much more radio emission inside the source and confirmed its complicated radio structure. We suggest there are a strong jet-cloud interactions present in that source and changing the jet path. However not all the features visible on the new radio images can be explained by the interactions with the surrounding medium. We suspect there is a jet precession persent in that source, and the detailed discussion will be presented in a forthcoming paper.

\section{Acknowledgement}

MERLIN is a UK National Facility operated by the University of Manchester on behalf of STFC. The European VLBI Network is a joint facility of European, Chinese, South African and other radio astronomy institutes funded by their national research councils.

This work was supported by Polish Ministry of Science and Higher Education under grant N N203 303635. Partial support for this work was provided by the UMK under grant no. 410-A.

\section{References}

[1] Becker, R. H., White, R. L., Gregg, M. D., et al., Properties of Radio-selected Broad Absorption Line Quasars from the First Bright Quasar Survey, 2000, ApJ, 538, 72

[2] Elvis, M.,A Structure for Quasars, 2000, ApJ, 545, 63

[3] Gregg, M. D., Becker, R. H., Brotherton, M. S., et al., Discovery of a Classic FR II Broad Absorption Line Quasar from the FIRST Survey, 2000, ApJ, 544, 142

[4] Jiang, D. R. \& Wang, T. G., EVN observation of three Broad Absorption Line quasars, 2003, A\&A, 397, L13

[5] Kunert-Bajraszewska, M., \& Marecki, A., FIRST-based survey of compact steep spectrum sources. V. Milliarcsecond-scale morphology of CSS objects, 2007, A\&A, 469, 437

[6] Kunert, M., Marecki, A., Spencer, R. E., Kus, A. J., \& Niezgoda J., FIRST-based survey of Compact Steep Spectrum sources. I. MERLIN images of arc-second scale objects, 2002, A\&A, 391, 47

[7] Liu, Y., Jiang, D. R., Wang, T. G. \& Xie, F. G, The compact structure of radio-loud broad absorption line quasars, 2008, MNRAS, in print

[8] White, R. L., Becker, R. H., Helfand, D. J., \& Gregg, M. D., A Catalog of 1.4 GHz Radio Sources from the FIRST Survey, 1997, ApJ, 475, 479

[9] Willott, C. J., Rawlings, S., Archibald, E. N., \& Dunlop, J. S., A submillimetre difference between radio galaxies and radio quasars: evidence for quasar-heated dust and synchronized submillimetre and radio source activity, 2002, MNRAS, 331, 435 